\title{
Clinical guidelines
}

\section{Summary}

Greater understanding of the underlying pathophysiology of acute myocardial infarction (AMI) has led to more aggressive management and lower mortality, both in-hospital and long term. AMI results mainly from thrombotic occlusion of the infarct-related coronary artery. The ensuing necrosis evolves over a 6-12 h period providing a time window for interventions designed to reduce eventual infarct size. The most appropriate interventions are those which restore coronary artery patency and hence myocardial blood flow as soon as possible. Occasionally, disruption of the occluding thrombus and compression of the underlying atheromatous lesion is best achieved by direct percutaneous transluminal coronary angioplasty. For the vast majority however, revascularisation by drug therapy is more appropiriate. As soon as possible, all patients without contraindications should be offered oral aspirin and intravenous thrombolysis, usually with streptokinase but occasionally with tissue plasminogen activator. Patients in whom these agents are contraindicated should be considered for intravenous betablockade using atenolol or metoprolol to reduce myocardial demand and hence infarct size. Patients with large infarcts, impaired ventricular function, left ventricular failure or hypertension should be considered for early angiotensin-converting enzyme inhibitor therapy. Other agents may be valuable symptomatically, but have no proven role in reducing infarct size or mortality. After the first $24 \mathrm{~h}$, the main aims of management are to assess the likelihood of later ischaemic events or death (risk stratification) and hence to choose appropriate long term secondary prophylaxis.

Keywords: acute myocardial infarction, clinical guidelines, thrombolysis

Royal Liverpool University Hospital Trust, Prescot Street, Liverpool

L7 8XP, UK

S Saltissi

SS Mushahwar

Accepted 19 April 1995

\section{The management of acute myocardial infarction}

\section{S Saltissi, SS Mushahwar}

Recently, radical advances in the management of acute myocardial infarction (AMI) have resulted in a major reduction in early mortality from $10-15 \%$ in the pre-thrombolytic era to approximately $5-10 \%$ today. ${ }^{1}$

The impetus for these improvements has come largely from improved understanding of the pathophysiology of AMI. It is now clear that AMI almost always results from total occlusion of a coronary artery by thrombus, usually at the site of a recently cracked or fissured atheromatous plaque (figure 1). Superimposed spasm may play a role whilst other pathologies, eg, embolism, arteritis, are much rarer.

Infarction does not occur instantaneously following this thrombosis. Cells progress from reversible to irreversible damage over several hours causing

\section{UNCOMPLICATED PLAQUE}

No symptoms or stable angina.

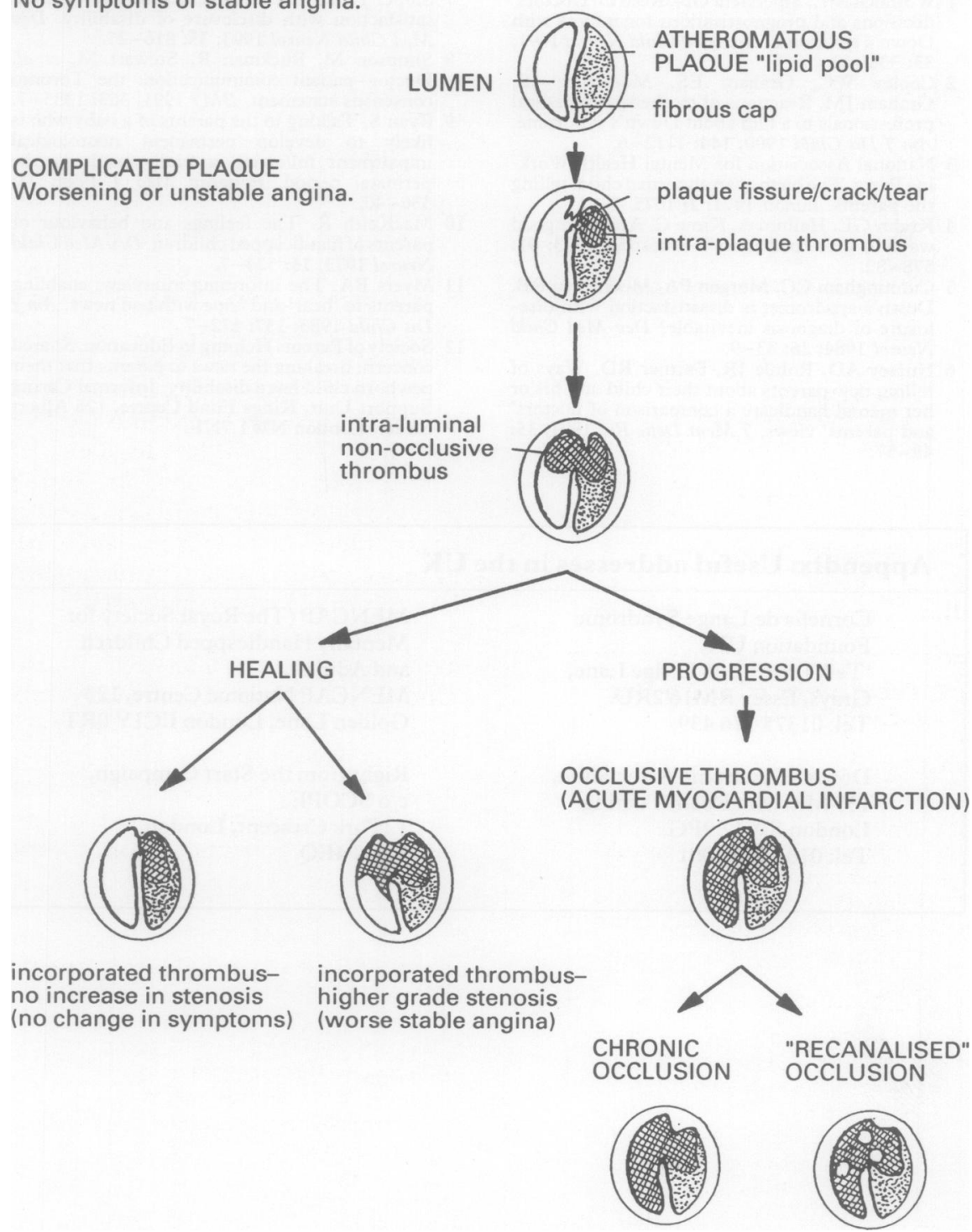

Figure 1 Temporal progression of atheromatous plaque and clinical outcome 


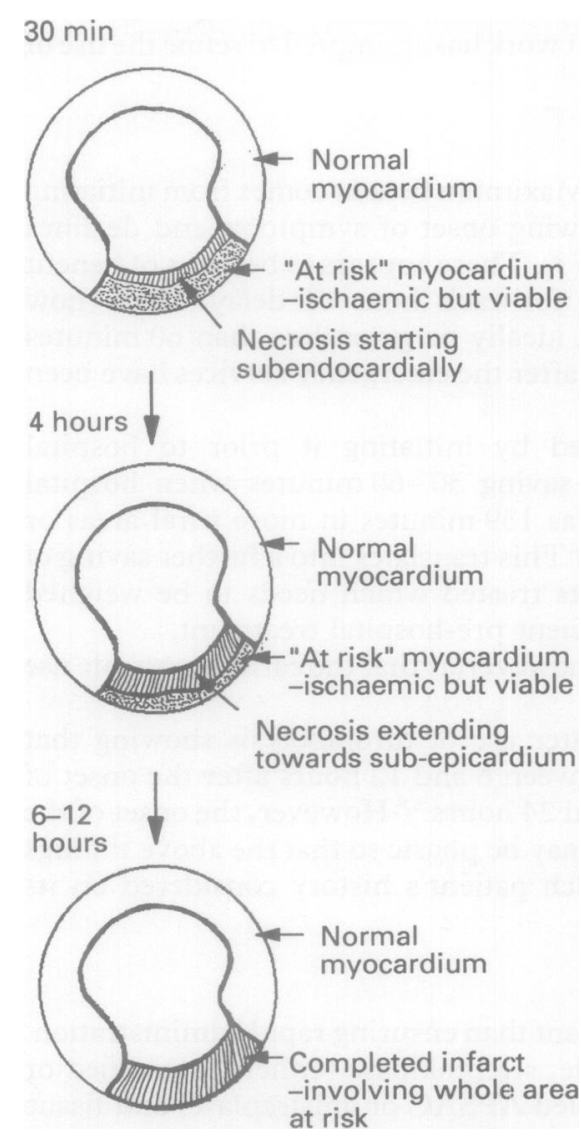

Figure 2 Progression of necrosis in AMItime dependency. Transverse sections of the left ventricle after occlusion of a coronary artery

\begin{tabular}{|l|}
\hline Strategy for the manage- \\
ment of AMI \\
\hline treatment of the acute attack \\
(0-24 h) \\
- symptomatic relief \\
- modification of the infarction \\
process \\
- risk stratification ( $1-6$ weeks $)$ \\
- secondary prevention (1 week \\
onwards)
\end{tabular}

Box 1

\section{Treatment of the acute} attack

- symptomatic relief

- analgesia (opiates \pm anti-emetic)

- oxygen

- nitrates (sublingual, buccal, intravenous)

- infarct modification

- improve blood supply

- thrombolysis (streptokinase or

tPA)

- aspirin

- primary (direct) PTCA

- reduce oxygen demand beta-blockade (atenolol, metoprolol)

- alter early remodelling - ACE inhibitor therapy (captopril, ramipril, lisinopril) necrosis to spread like an advancing wavefront through the 'at risk' myocardial region supplied by the thrombosed coronary artery (figure 2). Unchecked, infarction usually reaches its maximal extent by 6-12 hours. Salvage of myocardium destined to die and hence reduction in infarct size can be achieved during this six-hour time window using several different therapeutic interventions. However, by far the most effective is to restore coronary artery patency and hence achieve reperfusion of the ischaemic muscle as soon as possible.

In the hours and days following AMI, important changes occur in the infarct itself (thinning, expansion) as well as in the rest of the left ventricle (compensatory hypertrophy, dilatation). Together these changes have been termed remodelling. Although occasionally beneficial, they are often deleterious and may constitute the first steps in the spiral towards decreasing left ventricular function and heart failure. Fortunately this remodelling process is also now amenable to therapeutic modification.

Death during the first few minutes or hours following AMI is predominantly due to an arrhythmia (ventricular fibrillation) whereas in the later hospital phase pump failure predominates. Patients who survive to leave hospital are not 'out of the woods' but remain at increased risk due to further ischaemic events or worsening left ventricular function consequent upon deleterious remodelling.

\section{Current strategy for the management of AMI}

Modern management of AMI can be divided into three somewhat overlapping stages (box 1). During the first 24 hours the principal aim is to deal with the acute attack itself after which attention is directed to assessing the risks of future ischaemic problems (risk stratification) and thence to strategies designed to reduce that risk (secondary prevention). This article will concentrate on treatment of the acute attack, ie, within the first 24 hours.

\section{Treatment of the acute attack (box 2)}

Cardiac pain is due to continuing ischaemia of jeopardised but viable myocardium. Pain relief should be initiated by sublingual glyceryl trinitrate either as spray or tablets which can then be followed by buccal nitrate to cover patient transfer. Continuing pain requires opiates such as morphine sulphate or diamorphine, 2.5 to $5 \mathrm{mg}$ intravenously, repeated every 15-30 minutes as necessary providing there are no contraindications, eg, chronic obstructive airways disease. Naloxone (Narcan 0.4-2.0 mg intravenously) should always be available and cyclizine should be given with the opiate. Additional benefits of opiates include an anxiolytic effect together with both vaso- and veno-dilatation which reduce cardiac work.

As soon as possible attention should be turned to instituting ways of modifying the infarct process to minimise left ventricular damage during the all-important early hours. Over the last decade a plethora of potential treatments have appeared which, used indiscriminantly, could produce both confusion and inappropriate polypharmacy. In the following section, the place of each potential treatment will be considered but it is important to bear in mind that the main goal of treatment is to restore perfusion by opening up and maintaining patency of the thrombosed infarct-related coronary vessel.

\section{THROMBOLYTIC THERAPY}

Undoubtedly, the advent of thrombolysis has revolutionised the treatment of AMI. Several large scale studies ${ }^{2-5}$ have established that the early use of thrombolysis reduces overall mortality by around $20 \%$ (table) and preserves myocardium by dissolving intracoronary clot (so restoring patency) in about $55-75 \%$ of patients. Thrombolysis should therefore be initiated as soon as possible in all patients without contraindications presenting within 12 hours of a

Table Relative benefits of treatments for myocardial infarction ${ }^{8}$

\begin{tabular}{ll}
\hline Treatment & Lives saved per 1000 patients treated \\
\hline Intravenous thrombolysis & \\
$\bullet$ streptokinase pre-hospital & $35-40$ \\
streptokinase in-hospital & $20-25$ \\
tPA (alteplase) & 35 \\
Oral aspirin & $20-25$ \\
Oral ACE inhibitor & $5-8$ \\
Intravenous beta-blocker & 6 \\
\hline
\end{tabular}




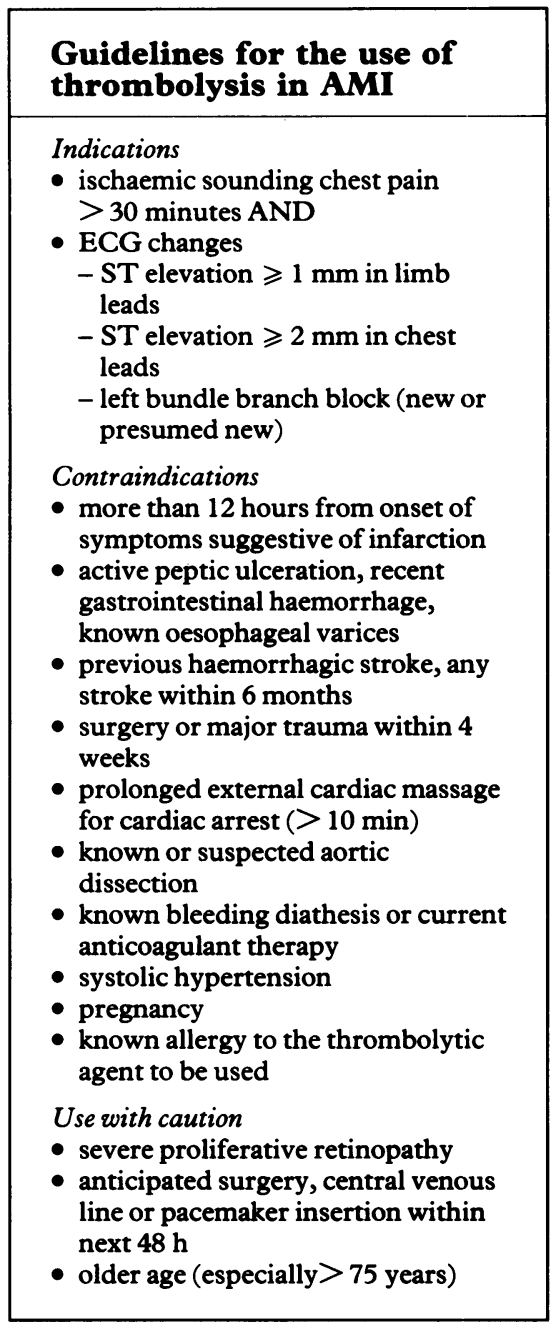

Box 3

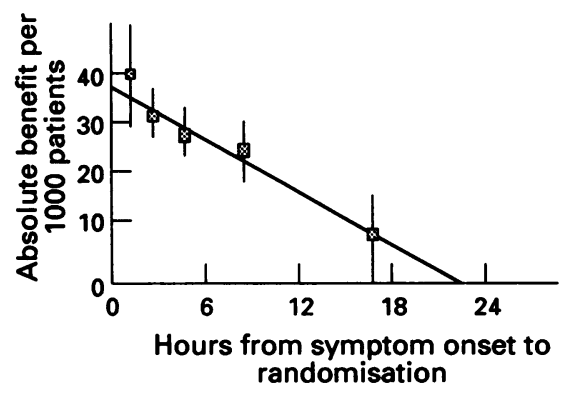

Figure 3 Time-dependent benefits of thrombolysis. Graph shows absolute reduction in mortality at 35 days $v$ s delay from onset of symptoms to randomisation among 45000 patients with ST elevation or bundle branch block. Modified from FTT Collaborative Group. ${ }^{6}$ suspected AMI (box 3). Much subsequent work has attempted to refine the use of thrombolytic agents in AMI as follows:

\section{Timing}

This is the single most important factor. Maximum benefit comes from initiating thrombolysis within the first hour following onset of symptoms and declines exponentially with time thereafter (figure 3). There appears to be a loss of benefit of 1.6 lives per 1000 patients treated for each hour of delay. ${ }^{6}$ It is now recommended that thrombolysis should ideally be given less than 60 minutes and in practice no more than 90 minutes after the emergency services have been called.

Earlier thrombolysis can be achieved by initiating it prior to hospital admission (usually using anistreplase), saving 30-60 minutes when hospital administration is efficient and as much as 139 minutes in more rural areas or when hospital administration is delayed. ${ }^{8}$ This translates into a further saving of approximately 20 lives per 1000 patients treated which needs to be weighed against the difficulties of organising efficient pre-hospital treatment.

The clear message from all the above, however, is that the earliest possible use of thrombolysis is essential.

Several studies have addressed the later use of thrombolysis showing that significant benefit can still be shown between 6 and 12 hours after the onset of symptoms but little if any between 12 and 24 hours. ${ }^{6,8}$ However, the onset of the infarction process is often not clear and may be phasic so that the above timings should not be taken as absolute and each patient's history considered on its merits.

\section{Agent}

The choice of thrombolytic is less important than ensuring rapid administration. Direct comparison of the main agents, ie, streptokinase (either unmodified or when part of an anisoylated complex called APSAC or anistreplase) and tissue plasminogen activator (tPA or altephase) has shown no significant advantage for any agent. ${ }^{9}$ However the subcutaneous heparin regime used may have reduced the potential benefit of tPA and the subsequent GUSTO study ${ }^{10}$ using intravenous heparin demonstrated a $1 \%$ further reduction in absolute mortality in favour of an accelerated tPA regime compared to intravenous streptokinase. Sub-group analysis showed this benefit to be particularly prominent in patients under the age of 75 years presenting within four hours of an anterior infarct. This has to be balanced, however, against significantly greater costs $(8-10$ fold), a higher incidence of haemorrhagic strokes $(0.5 \%$ for streptokinase versus $0.7 \%$ for tPA) especially in the over 75-year-olds, and a more complex administration schedule.

A limiting factor in the use of streptokinase in either of its forms, is the fact that it is antigenic, with the development of significant neutralising antibody titres starting five days after exposure. Repeated use within a year must therefore be avoided, ${ }^{11}$ whilst the continuing presence of significant antibody titres as long as four years after initial exposure ${ }^{12}$ suggests that its reuse may need to be avoided for many years and perhaps for ever.

In view of the above, it is recommended that the standard thrombolytic agent used should be streptokinase (box 4). In certain subgroups of patients (box 5) it is preferable to use tPA via its accelerated (front loaded) schedule (box 4). There is currently no definite place for anistreplase though its simple bolus administration may make it suited for out-of-hospital administration.

\section{Heparin}

The use of tPA must be accompanied by intravenous heparin (box 4) whereas neither subcutaneous nor intravenous heparin adds anything to the efficacy of streptokinase. Subcutaneous herapin should continue to be used in low dose for the prevention of deep vein thrombosis or in higher dose (12 500 units bid) to prevent mural thrombus and systemic embolisation following anterior infarction. ${ }^{13}$

\section{Routine post-thrombolysis angioplasty}

Although thrombolysis restores anterograde perfusion in $75 \%$ of patients, many will have residual severe or thrombogenic stenoses which may lead to recurrent thrombotic occlusion. Percutaneous transluminal coronary angioplasty (PTCA) has therefore been suggested as a means of reducing the severity of residual stenoses and hence the likelihood of recurrent occlusion (figure 4). However, several trials have demonstrated that PTCA performed routinely immediately after intravenous thrombolytic therapy is not beneficial and may actually be deleterious. ${ }^{14}$ Similarly, the strategy of routine deferred PTCA $18-48$ hours 


\begin{tabular}{|l|}
\hline Thrombolytic drug \\
regimens based on use in \\
clinical trials \\
\hline Streptokinase (ISIS-3) \\
- 1.5 million units as slow \\
intravenous infusion over $1 \mathrm{~h}$ \\
- no need for intravenous heparin \\
tPA, alteplase (GUSTO) \\
- $15 \mathrm{mg}$ bolus then, \\
$0.75 \mathrm{mg} / \mathrm{kg}$ (max $50 \mathrm{mg}$ ) over \\
$30 \mathrm{~min}$ then, \\
- $0.5 \mathrm{mg} / \mathrm{kg}$ (max $35 \mathrm{mg}$ ) over $60 \mathrm{~min}$ \\
intravenous heparin -5000 units \\
bolus then infusion for $48 \mathrm{~h}$ (APTT \\
$1.5-2.5$ control) \\
Anisoylated plasminogen activator \\
complex - APSAC, anistreplase \\
(ISIS-3) \\
- $30 \mathrm{mg}$ intravenous over 3 min \\
(suitable for pre-hospital \\
treatment) \\
- no need for intravenous heparin \\
\end{tabular}

Box 4

Indications for tPA therapy
Accepted
- known allergy to streptokinase
- previous streptokinase use within 1
year
- known recent streptococcal
infection
Possible (accepted by some
authorities)
- patients under 75 years with
anterior MI within $4 \mathrm{~h}$ of onset
- hypotension (systolic blood
pressure $<100$ mmHg) during
streptokinase infusion
- previous streptokinase use at any
time

Box 5

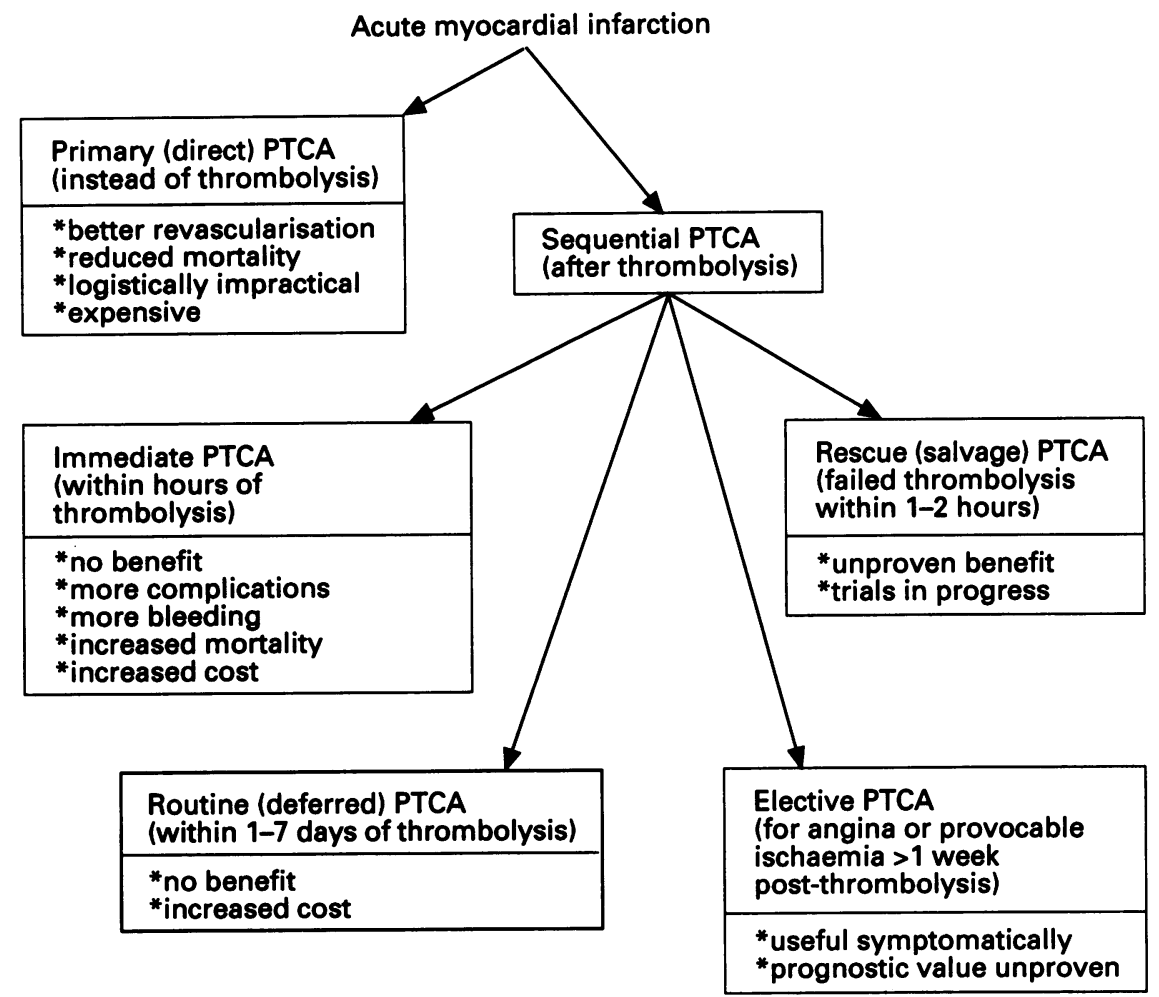

Figure 4 PTCA and AMI

after thrombolysis does not improve left ventricular function or survival. ${ }^{14}$ PTCA following thrombolysis should therefore be reserved for those patients with symptomatic or objective evidence of ischaemia.

\section{ASPIRIN}

Platelets are intimately involved in the initial formation of a thrombus at the site of a ruptured atheromatous plaque. Even if lysis of a thrombus can be induced, the underlying stimuli to platelet-initiated rethrombosis persist for many hours or longer. Aspirin has inhibitory effects on platelets because it suppresses the enzyme cyclo-oxygenase which is necessary for the synthesis of certain prostenoids (particularly thromboxane A2). It is thus a logical agent to try and delay or prevent re-occlusion after successful lysis.

Its clinical efficacy has now been established in several studies but particularly ISIS- $2^{5}$ in which patients receiving aspirin alone had a $23 \%$ reduction in mortality compared to a $25 \%$ reduction in those treated with intravenous streptokinase alone (table). Importantly, in the group receiving both agents, there was an additive benefit leading to a $42 \%$ reduction in mortality compared to the group with neither agent. In addition, non-fatal strokes and reinfarctions were reduced in the aspirin-treated group.

Since aspirin is cheap and relatively safe, $150-300 \mathrm{mg}$ should be given to all patients with suspected AMI as soon as possible unless definite contraindications exist, such as known aspirin intolerance, active peptic ulcer disease, or recent gastro-intestinal bleeding.

The relative efficacy of aspirin, warfarin or placebo after angiographically successful thrombolysis has been studied. ${ }^{8}$ Aspirin proved superior to both placebo and warfarin in preventing clinical events and preserving ventricular function but was no better than warfarin at reducing reinfarction or the need for revascularisation (both were better than placebo). Angiographic coronary re-occlusion was not significantly prevented by either agent. This area remains to be clarified and ongoing studies are awaited.

\section{BETA-BLOCKER THERAPY}

Apart from thrombolytic agents and aspirin, the only other treatment consistently shown to improve outcome when administered acutely is intravenous beta-blockade using either metoprolol or atenolol. ${ }^{15}$ The reduced mortality has been attributed largely to a reduction in cardiac rupture rather than to other possibilities such as reduced arrhythmic events or reinfarction rates. These studies were, however, done in the pre-thrombolytic era and whether the $15 \%$ reduction in mortality achieved (table) would be additional to that obtained using 
thrombolysis is currently unknown. Nonetheless, they did show that intravenous beta-blockade is a very safe therapy provided inappropriate patients are excluded, eg, those with severe braycardia, marked hypotension, heart failure or cardiogenic shock.

We recommend that intravenous atenolol $(5 \mathrm{mg}$ bolus over five minutes followed by further $5 \mathrm{mg}$ bolus over the next five minutes if the heart rate is greater than 80 beats per minute, followed by oral atenolol) be given to suitable patients with contraindications to thrombolysis. It should also be considered in addition to thrombolysis in those patients with persistent hypertension or inappropriate tachycardia. ${ }^{11}$

\section{ANGIOTENSIN-CONVERTING ENZYME (ACE) INHIBITORS}

These drugs have emerged as being of major benefit in the acute phase of myocardial infarction. Initially, fears about the safety of ACE inhibitor therapy early after infarction arose out of the CONSENSUS II study ${ }^{16}$ of intravenous enalapril given within $24 \mathrm{~h}$ of acute infarction in which mortality was higher in the treated group. Subsequently, however, GISSI $3^{17}$ using lisinopril, and ISIS $4^{18}$ and $\mathrm{CCS}^{19}$ using captopril established both the safety of the drugs, and also their efficacy in a broad range of patients when begun $<36 \mathrm{~h}$ post-AMI and continued for 4-6 weeks. Taken together, these trials indicate a reduction in early (4-6 week) mortality of about 7\% equating to five lives saved per 1000 patients treated. The survival advantage appeared to be maintained unchanged in the longer term (one year).

In a further recent trial (SMILE ${ }^{20}$ ) an ACE inhibitor, zofenopril, was studied in patients with anterior infarction not undergoing thrombolysis. In this high-risk cohort, short-term treatment (six weeks) not only improved both mortality (by $25 \%$ ) and the risk of severe congestive heart failure (by $46 \%$ ) in the short term but also at one year (mortality reduced by $29 \%$ ).

The only significant adverse effect of early ACE inhibitor therapy in these studies was a consistent but small increase in hypotension, severe enough to require cessation of therapy.

From the above, the emerging belief is that early ACE-inhibitor treatment favourably alters left ventricular remodelling so reducing dilatation, improving contractile function and hence both short- and long-term outcome. A separate series of trials using ACE inhibitor therapy later (three days onwards) (to be discussed in a future article) has also established ACE inhibitors as very effective agents for secondary prophylaxis after infarction.

How best to use this trial data in clinical practice is not yet clear. One logical strategy is to start ACE inhibitor therapy from day one onwards in all patients without contraindications or sustained hypotension (systolic blood pressure $<100 \mathrm{mmHg}$ ) but particularly in large and especially anterior infarctions. Treatment should then stop after 4-6 weeks except where clinical left ventricular impairment is present or asymptomatic reduction in ventricular function (ejection fraction $<40 \%$ ) can be demonstrated, when treatment should be continued long term.

\section{OTHER POTENTIAL THERAPIES}

\section{Calcium channel antagonists}

On theoretical grounds and in the experimental model, calcium antagonists are useful in reducing myocardial necrosis following coronary artery occlusion and reperfusion. The expectation, therefore, was that they would also be useful in the setting of AMI but, unfortunately, many trials using different drugs have proved either neutral or, particularly with dihydropyridine agents, have actually suggested detriment. The latest such study (SPRINT 2) ${ }^{21}$ showed that nifedipine $60 \mathrm{mg}$ per day started within 48 hours of admission with AMI resulted in a higher mortality $(15.4 \%)$ than with placebo $(13.3 \%)$ though the difference was not significant. The message, however, is clear. There is no place for calcium antagonists in the acute management of myocardial infarction other than to treat post-MI angina or hypertension when a beta-blocker cannot be used.

\section{Nitrates}

In recent years the use of nitrates in the setting of AMI has become widespread, either in the form of sublingual tablets or spray, buccal tablets, or intravenous infusion. The theoretical grounds for expecting beneficial effects on the infarcting process are sound, and meta-analysis of many small studies suggested a potentially very large reduction in death and re-infarction. Unfortunately, when the hypothesis was tested prospectively in two of the largest cardiovascular 
trials ever - the ISIS 4 study ${ }^{18}$ and GISSI $3,{ }^{17}$ no significant effect on mortality was found using either transdermal patches or long-acting tablets. However, no detrimental effect emerged.

The current status of nitrates in AMI is therefore that they have no place as disease-modifying agents but that they are very useful symptomatically for myocardial ischaemic pain due to the infarct itself and to post-infarction angina or alternatively they are useful for dyspnoea due to left ventricular dysfunction.

\section{Magnesium}

In the mid 1980s enthusiasm grew for the use of intravenous magnesium in the early phase of myocardial infarction. Its mechanism in this setting was not clear since arrhythmic deaths in hospital post-AMI are relatively few. However, in pharmacological doses, magnesium is known to produce coronary and systemic vasodilatation, platelet inhibition and may also reduce injury-mediated calcium influx, so helping to limit infarct size.

An overview of all studies up to 1990 involving 1300 patients showed significant reductions in both mortality and arrhythmias. ${ }^{22}$ A subsequent trial of intravenous magnesium (LIMIT-2) ${ }^{23}$ in over 2000 patients showed a $24 \%$ reduction in 20-day mortality independent of thrombolytic therapy. However, in the very large ISIS 4 study, ${ }^{18}$ magnesium proved to have no benefit as a primary therapeutic agent in AMI.

In the light of these conflicting results the use of magnesium remains controversial. The difference between the results of the two trials may be accounted for by the relatively late administration of magnesium in ISIS 4 as opposed to its use at or around the time of thrombolysis in LIMIT-2. For the moment, however, routine use of magnesium in AMI cannot be justified.

\section{Anti-arrhythmic therapy}

Ventricular fibrillation is the main cause of death in the first few hours of AMI. The concept that lesser ventricular arrhythmias (frequent, multiform or R-on-T ectopics; unsustained ventricular tachycardia) might warn of impending ventricular fibrillation, ${ }^{24}$ led to the establishment of coronary care units for continuous electrocardiogram monitoring. However, many studies within the community and in coronary care units have assessed the value of prophylactic anti-arrhythmic drugs (mainly lignocaine) in suppressing these warning arrhythmias and hence in preventing ventricular filbrillation. An overview of these studies $^{25}$ has not shown any significant benefit in terms of reducing mortality although suppression of ventricular ectopic activity was effectively achieved. Intravenous lignocaine should therefore not be used prophylactically but is routinely used to prevent recurrence of ventricular fibrillation after an initial event.

\section{Primary PTCA for acute myocardial infarction}

Definition

- PTCA as first line treatment, no associated thrombolysis

\section{Advantages}

- $>90 \%$ coronary patency rates

- both thrombus and underlying stenosis treated

- improved mortality

- no major bleeding problems (cerebral or gastrointestinal)

\section{Disadvantages}

- expensive

- requires 24 h availability of catheter lab

- requires $24 \mathrm{~h}$ availability of experienced interventional cardiologists

- $13 \%$ re-occlusion rate

Current indications (assuming rapid availability of cardiac catheter lab with trained staff

- when thrombolysis

contraindicated, eg, peptic ulcer hypotension, etc.

- cardiogenic shock early after extensive AMI

\section{NON-PHARMACOLOGICAL THERAPIES}

The realisation that the overriding need following AMI is to re-establish coronary artery flow to the affected region has led to evaluation of direct interventional methods. Coronary artery bypass grafting has been assessed as an immediate therapy in AMI, but it is impractical and expensive and is therefore not used as a routine treatment option in the UK (though occasionally it is in the US).

The technique of primary PTCA to the occluded infarct-related vessel (box 6, figure 4) was first introduced in $1983^{26,27}$ as a means of restoring flow without the need for thrombolytic therapy and its haemorrhagic complications. It causes mechanical disruption of the occlusive thrombus and the underlying stenosis in one sitting, so rapidly restoring coronary flow. Patency rates of the order of $90 \%$ are obtained compared to those of $55-75 \%$ resulting from thrombolysis. Several recent studies have compared these two treatment modalities. The largest of these, the PAMI study ${ }^{28}$ showed a lower in-hospital mortality rate ( 2.6 vs $\left.6.5 \%\right)$, lower stroke rate ( 0 vs $3.5 \%$ ), lower recurrent ischaemia rate $(10.3$ vs $28 \%$ ) and a shorter in-hospital stay (7.5 vs 8.4 days) with direct PTCA. These studies collectively suggest that primary PTCA may well be clinically more effective than thrombolysis. However the disadvantages of this technique (box 6) mean that its widespread adoption as a routine treatment in AMI is impractical.

\section{Conclusion}

The management of AMI is now regarded as a series of stages starting with the treatment of the acute attack which attempts both to relieve symptoms and to modify the disease process. The primacy of the restoration of flow down the infarct-related vessel has been emphasised and the roles of a number of pharmacological agents as well as PTCA discussed. Figure 5 summarises our 


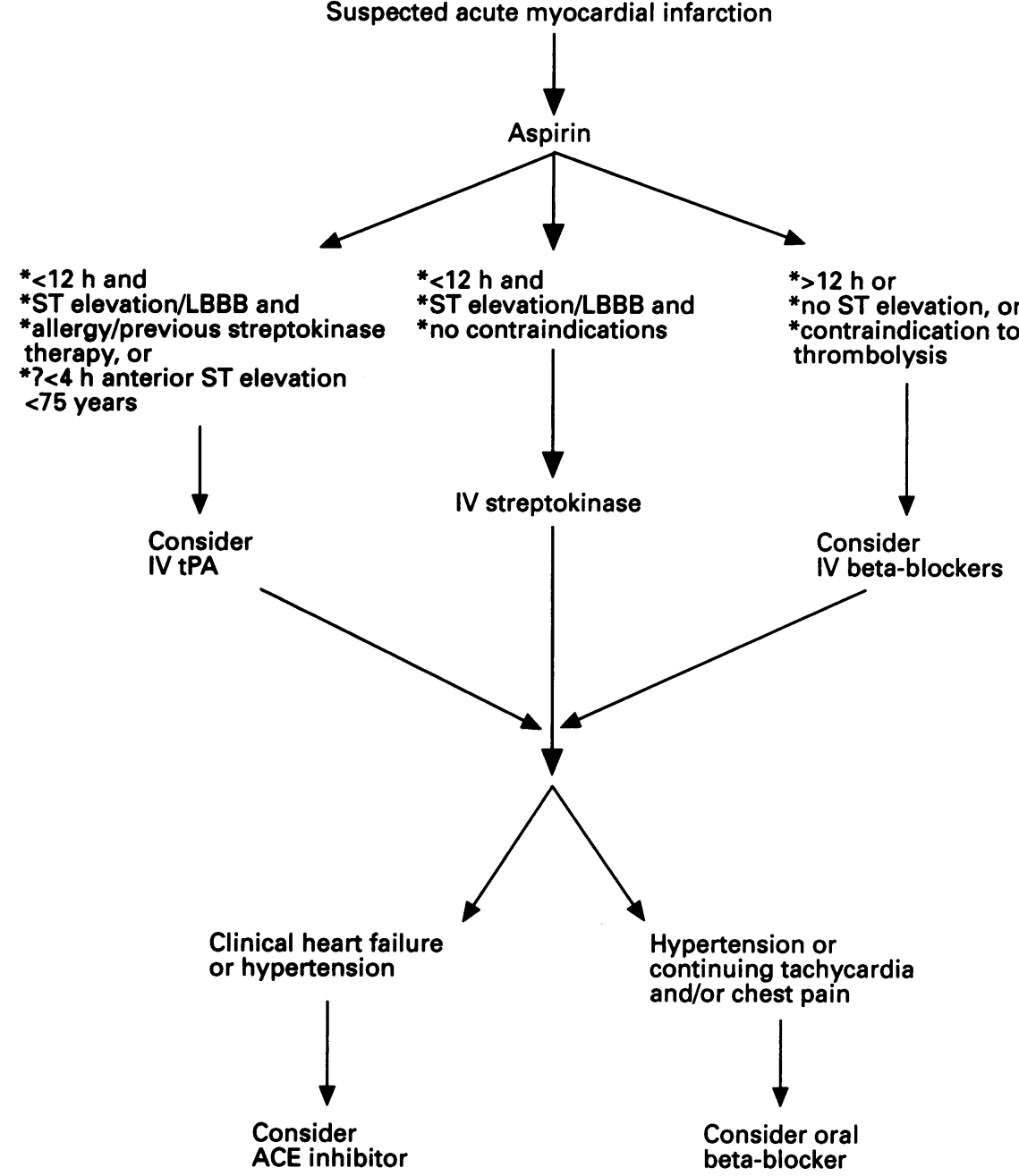

Figure 5 Treatment of AMI during the first $24 \mathrm{~h}$ - a summary algorithm

approach to the management of AMI during the first $24 \mathrm{~h}$ whilst the table compares the likely benefits to be achieved by the individual agents in terms of the number of lives saved.

Once the time window for infarct modification has passed (usually within the first $24 \mathrm{~h}$ ) attention should then be diverted to risk stratification and secondary prevention, which will constitute the basis of a separate review article.

1 Yusuf S, Sleight P, Held P, MacMahon S Routine medical management of acute myocardial infarction: lessons from overviews of recent randomised controlled trials. Circulation 1990; 82 (suppl II): II-117-34.

2 Gruppo Italiano per lo Studio della Streptochinasi nell 'Infarcto Miocardico (GISSI). Effectiveness of intravenous thrombolytic treatment in acute myocardial infarction. Lancet ment in acute my $397-401$.

3 ISIS-2 (Second International Study of Infarct Survival) Collaborative Group. Randomised Survival) Collaborative Group. Randomised trial of intravenous streptokinase, oral aspirin, both or neither among 17,187 cases of suspected acute myocardial

4 De Bono DP. Coronary thrombolysis. Br Heart f 1987; 57: 301-5.

5 Anderson HV, Willerson JT. Thrombolysis in acute myocardial infarction. N Engl $\mathcal{F}$ Med 1994; 329: 703-9.

6 Fibrinolytic Therapy Trialists (FTT) Collaborative Group. Indications for fibrinolytic therapy in suspected acute myocardial infarction: collaborative overview of early mortality and major morbidity results from all randomised trials of more than 1000 patients. Lancet 1994; 343: 311-22.

7 Weston CFM, Penny WJ, Julian DG, on behalf of the British Heart Foundation Working Group. Guidelines for the early management of patients with myocardial infarction. BMF 1994; patients with $767-71$.
8 McMurray J, Rankin A. Cardiology-I: treatment of myocardial infarction, unstable angina, and angina pectoris. BMF 1994; 309: 1343-50 9 ISIS-3 (Third International Study of Infarc Survival) Collaborative Group. ISIS-3: a randomised comparison of streptokinase vs tissue plasminogen activator vs anistreplase and of aspirin plus heparin vs aspirin alone amon 41,299 cases of suspected acute myocardial infarction. Lancet 1993; 339: 753-70.

10 The GUSTO Investigators. An international randomised trial comparing four thrombolytic randomised trial comparing four thrombolytic strategies for acute myocardial

11 De Bono DP, Hopkins A. The management of acute myocardial infarction: guidelines and audit standards. $\Im R$ Coll Physicians Lond 1994 28: 312-7.

12 Elliot JM, Cross DB, Cederholm-Williams S, White HD. Streptokinase titres 1 to 4 years after intravenous streptokinase. Circulation 1991; 84, 111-6.

13 Turpie AGG, Robinson JG, Doyle DJ, et al. Comparison of high-dose with low-dose subcutaneous heparin to prevent left ventricular mural thrombus in patients with acute transmural anterior myocardial infarction. $N$ Engl $f$ Med 1989; 320: 352-7.

14 Landau C, Lange RA, Hillis LD. Percutaneous transluminal coronary angioplasty. $N$ Engl $f$ Med 1994; 330: 981-93.
15 ISIS-1 (First International Study of Infarct Survival) Collaborative Group. Randomised trial of intravenous atenolol among 16,027 cases of suspected acute myocardial infarction: ISIS1. Lancet 1986; ii: 57-66.

16 Swedberg K, Held P, Kjekshus J, et al on behalf of the CONSENSUS II study group. Effects of the early administration of enalapril on mortality in patients with acute myocardial infarction. in patients with acute myocardial infarction Results of the Co-operative New Scandinavian II). $N$ Engl $f$ Med 1992; 327: 678-84.

17 Gruppo Italiano per lo Studio dela Sorpavivenza Gruppo Italiano per lo Studio dela Sorpavivenza nell-Infarcto Miocardico. GISSI-3: effects of lisinopril and transdermal glyceryl trinitrate singly and together on 6-week mortality and ventricular function after acute myocardia infarction. Lancet 1994; 343: 1115-22.

18 ISIS-4 (Fourth International Study of Infarct Survival) Collaborative Group. A randomised factorial trial assessing early oral captopril, oral mononitrate, and intravenous magnesium sulphate in 58,050 patients with suspected acute myocardial infarction. Lancet 1995; 345: 669-85.

19 Chinese Cardiac Study Collaborative Group Oral captopril versus placebo among 13,634 patients with suspected acute myocardial infarction: interim report from the Chinese Cardiac Study (CCS-1). Lancet 1995; 345: 868-87. 
20 Ambrosioni E, Borghi C, Magnani B, for the Survival of Myocardial Infarction Long-Term Evaluation (SMILE) Study Investigators. The effect of the angiotensin-converting enzyme inhibitor zofenopril on mortality and morbidity after anterior myocardial infarction. $N$ Engl $f$ Med 1995; 332: 80-5.

21 Gouldbourt U, Behar S, Reicher-Reiss H, Zion M, Mandelze in suspected acute ministration of nifedipine Intern Med 1993; 153: 345-53. 22 Teo KK, Yusuf S, Collins R, Held PH, Peto R. Effects of intravenous magnesium in suspected acute myocardial infarction: overview of ran-
domised trials. $B M \Im$ 1991; 303: 1499-1503.
23 Woods KL, Fletcher S, Roffe C, Haider Y Intravenous magnesium sulphate in suspected acute myocardial infarction: results of the second Leicester intravenous magnesium tria (LIMIT 2). Lancet 1992; 339: 553-8.

24 Lown B, Wolf $M$. Approaches to sudden death from coronary heart disease. Circulation 1971; 44: 130.

25 MacMahon S, Collins R, Peto RW, Yusuf A. Effects of prophylactic lignocaine in suspected acute myocardial infarction. An overview of results from the randomised clinical trials. fAMA 1988; 260: 1910-6.
26 Hartzler GO, Rutherford BD, McConahay DR, et al. Percutaneous transluminal coronary angioplasty with and without thrombolytic therapy for treatment of acute myocardial infarction. $A m$ Heart I 1983; 106: 765-73

27 Grech ED, Ramsdale DR. Percutaneous transluminal coronary angioplasty and acute myocardial infarction. Br $\mathcal{F}$ Hosp Med 1994; 52: 35-41.

28 Grines CL, Browne KF, Marco J, et al. A comparison of immediate angioplasty with thrombolytic therapy for acute myocardial infarction. N Engl $\Im$ Med 1993; 328: 673-9.

\section{Medical Anniversary \\ LEO BUERGER, 13 SEPTEMBER 1879}

Leo Buerger (1879-1943) was born in Vienna, Austria, but was brought up and made his home in New York City. He graduated from the College of Physicians and Surgeons, Columbia University, (1901) and became assistant pathologist at Mount Sinai Hospital (1904). In 1908 he wrote Thromboangiitis obliterans; a study of the vascular lesions leading to presenile spontaneous gangrene (Am J Med Sci 1908; 136: 567).

He assisted in the development of a cystoscope and became professor of urology, at first in New York and then at the College of Medical Evangelists in Los Angeles. He died in New York on 6 October 1943. 